\title{
PRODUCCIÓN DE SUBJETIVIDAD A PARTIR DE LOS DISCURSOS GUBERNAMENTALES DEL ESTADO DE CHILE SOBRE LA DISCAPACIDAD
}

\section{Paula Serrano C. ${ }^{1}$}

\section{Resumen}

Las transformaciones de las últimas décadas en el ámbito productivo y social del Capital han reformado los procesos y prácticas institucionales de producción de subjetividad. La presente ponencia expone algunos de los resultados de una investigación que procura describir y analizar de forma exploratoria los procesos en que la discursividad del Estado de Chile desplegada entre los años 1992 y 2011 surge como territorio referencial para los sujetos, específicamente en el ámbito de aquellos cuerpos denominados discapacitados.

El estudio fue realizado entendiendo que dichos cambios se dan a partir de rupturas y continuidades que despliegan campos de inteligibilidad flexibles que gobiernan la vida de los sujetos. La noción de gubernamentalidad remite según Foucault al gobierno de la población a partir del ensamble de tecnologías de gobierno y racionalidades políticas en determinadas sociedades.

En el marco de los estudios sobre la gubernamentalidad, en este artículo presentamos algunos lineamientos que hemos identificado para comprender las nuevas lógicas de conducción de conducta y su constitución como políticas de intervención que modifican en un mismo movimiento la subjetividad y materialidad de los cuerpos clasificados y señalados como diferentes.

\section{Palabras claves:}

Gubernamentalidad, Producción de subjetividad, Discapacidad, Cuerpos, Saber/poder. 


\section{INTRODUCCIÓN}

El presente artículo expone los resultados de una investigación de tesis que abordó las tensiones que emergen en el campo de la producción de subjetividad en torno a las políticas enfocadas a cuerpos signados como discapacitados, tomando como eje de análisis la discursividad gubernamental del Estado de Chile publicada entre los años 1992 y 2011. Se propone reflexionar sobre la discapacidad, entendida como construcción social, y sus procesos de materialización, buscando desentrañar los poderes entramados en los mecanismos sociales de intervención del cuerpo y las subjetividades.

Considerando la complejidad de los procesos de constitución subjetiva y la imposibilidad de abarcarlos en su totalidad, la investigación fija un corte analítico, desde el cual se abordan sólo ciertos elementos involucrados. Se escogió al Estado de Chile y los discursos gubernamentales en torno a este como marco general de análisis. Así mismo, se demarcó un período de observación transversal que da cuenta de las continuidades y rupturas que conforman el proceso. Los años seleccionados surgen desde la recopilación de antecedentes, considerándose relevantes en tanto se inicia el 1992 con las primeras manifestaciones de la institucionalidad chilena sobre la necesidad de creación de un marco regulatorio para la integración de las personas con discapacidad y termina el año 2011 con la promulgación de la segunda ley que establece Normas sobre Igualdad de Oportunidades e Inclusión Social de las Personas con Discapacidad.

El Estado y su discursividad son las ventanas de acceso a los procesos de producción de subjetividad, sin embargo considerando la complejidad que enfrentamos a partir de las dinámicas diversificadas de valoración del Capital en su versión globalizada, se decidió estudiarlos desde el enfoque teórico gubernamental desarrollado por el autor M. Foucault. El Estado queda así planteado como eje de confluencia de poderes que encuentran su origen más allá de los límites del territorio del Estado-Nación. Su especificidad institucional será entendida como la autoridad política, desde la cual el "gobierno es codificado discursivamente, un modo de dividir la 'esfera política' a partir de la norma desde la cual las 'esferas no políticas' deben ser relatadas, y un modo para establecer temporal e institucionalmente tecnologías de gobierno" (Miller y Rose 1990: 177).

Para estudiar la discapacidad como un terreno de poder en disputa fue necesaria y pertinente una metodología que permitiera extraer de los documentos una organización analítica no lineal, ni evolutiva, sino que otorgara un marco explicativo de las formaciones discursivas, a través de la constitución del saber. Siguiendo este criterio, la perspectiva que se mostró como más eficaz es la genealogía Foucaultiana, la cual se ocupa del "análisis histórico de la producción de discursos y los modos en cómo funcionan en tanto que detonadores y sustentadores de relaciones de poder capaces de producir subjetividades" (Martínez, 2010: 74). En relación a esto, los conceptos genealógicos de Herkunft o procedencia y Entstehung o emergencia fueron claves para fijar líneas transversales de análisis en los discursos que permitieran comparación, como también un seguimiento de aquellos ejes conceptuales o ideas fuerza que desentrañen la red discursiva y su derivación en relaciones de saber/poder productoras de subjetividad.

Fueron seleccionados doce documentos que incluyeron tanto los discursos que conforman la gubernamentalidad del Estado de Chile, como la respuesta de algunas organizaciones de discapacitados que permitieran hacer un contraste relevante en términos analíticos. A partir de esto se operacionalizaron tres categorías teórico-metodológicas que enuncian y construyen una determinada Verdad sobre la discapacidad, unificando y articulando la interpelación fragmentaria a un determinado sujeto que se busca producir. Las categorías, que llamamos identificatorias, son las siguientes:

- Moral: esta categoría refiere al código o sistema ético que insta e incita a los sujetos a regularse en relación a él, estableciendo preceptos para 
conducir, juzgar su vida, y aceptar ciertas metas morales y expectativas para sí mismos. Es un medio efectivo para la regulación del yo, es decir para modular la conducción de conducta desde el propio individuo, esto se logra con la vinculación de su singularidad con objetivos morales respecto a las características deseables o no deseables para la gubernamentalidad.

- Democrática-institucional: esta categoría apela a la colectividad de la sociedad a partir de la ciudadanía, su participación, representación, derechos y deberes. Está directamente relacionada con la esfera del Estado, el ejercicio político, la administración jurídica y sus respectivas gestiones para fortalecer cierta racionalidad política.

- Tecno/ médica: esta categoría alude a la organización del cuerpo desde una perspectiva que no abarca solo la materialidad singular del individuo sino la relación de su cuerpo con las prótesis, el entorno y el saber generado sobre él. Involucra al dispositivo rehabilitación en el objetivo de potenciar o no ciertos órganos en pos de lograr determinadas habilidades que influenciarán en la calidad de vida del sujeto.

\section{Gubernamentalidad y los flujos de poder sobre el sujeto.}

Pensar la discapacidad como un fenómeno social nos remite necesariamente a considerar los procesos de exclusión e integración que se han desplegado sobre estos sujetos. Los antecedentes reunidos refieren a una historia, que denunciada primeramente desde la propia voz de los sujetos afectados y luego desde la discursividad de la institucionalidad, es definida por la frontera social de la opresión. Sin embargo, en el transcurso de las últimas décadas es posible presenciar el interés por parte de diferentes sectores sociales por revertir esta situación, proclamando un accionar tendiente a la incorporación de los sujetos denominados discapacitados a los ámbitos considerados normales por la sociedad. Tanto en el mundo como en Chile estos cambios se han sucedido y reflejado a partir de una serie de instancias que se pueden entender como campos estratégicos de poder, los cuales van constituyendo una forma de entender y de gestionar la discapacidad que va a situarse directamente en el cuerpo y la subjetividad misma de aquella persona considerada como discapacitada.

En relación a esta complejidad y la inscripción del poder sobre los cuerpos Scribano (2009) señala que "el capitalismo se ha transformado en una gran maquinaria depredatoria de energía -especialmente corporal -que ha trasformado, configurado- redefinido sus mecanismos de soportabilidad social y los dispositivos de regulación de las sensaciones" (p. 143).

En el caso de las personas con discapacidad, el anhelo de la integración articula un aparato de administración de los cuerpos vulnerables para imprimirles ciertas capacidades y habilidades necesarias para su desenvolvimiento en las relaciones que emergen de las transformaciones del capitalismo. La modulación de los cuerpos y los sujetos producida a partir de esta nueva realidad de superficies modificables y contingentes nos remite a la analítica del poder de Foucault. En este enfoque se sitúa al sujeto como atravesado y efecto del poder, observando su producción en el devenir de ciertas coordenadas históricas, lo cual facilita la observación contingente de su relación con el medio social. Los procesos de exclusión/ integración son parte de una estrategia de gobierno de la conducta en miras de estructurar el posible campo de acción de la población.

La consideración la población se origina en el interés de controlar los fenómenos que le son propios en función a los procesos económicos. Foucault (2000) Ilama a la intervención del poder en las fluctuaciones aleatorias y características biológicas de la población Biopolítica. El análisis del autor francés identifica los procesos de administración de la vida diferenciando dos momentos organizados en torno a la proximidad hacia una Norma. Primeramente, en las sociedades disciplinares del S. XVII, observa técnicas que buscan producir 
una subjetividad a partir del ajuste de los cuerpos a una Norma predeterminada del individuo deseable, es la llamada anatomopolítica. Las disciplinas gestionan la vida completa en función de enmarcar al sujeto en la relación tiempo-producción-costo transformándolo en fuerza de trabajo.

Posteriormente, Foucault (2004) observa una nueva forma de control a la que llama biopolítica. La mirada y el control son relocalizados hacia el del estudio de la población como agente con fenómenos aleatorios que le son propios. Se requiere regular la vida, haciéndola entrar en el dominio de los cálculos explícitos, intentando racionalizar los problemas relativos a la salud, la higiene, la natalidad, la longevidad, las razas y otros. La normalización para estos efectos no busca el ajuste de los cuerpos a una norma, sino que a partir de los fenómenos propios de la población se hace "interactuar esas diferentes distribuciones de normalidad y procurar que las más desfavorables se asimilen a las favorables" (p. 83).

Ante el escenario abierto por la problemática de la población, Foucault introduce el concepto de gubermentalidad, el cual le permite hacerse cargo del campo estratégico donde la actividad corporal es capturada en términos productivos por la relación capitalismo-sociedad liberal. La innovación que diseña el enfoque gubernamental presenta una entrada más eficaz a la dinámica de interacción de los elementos de las relaciones de poder, incluyendo una perspectiva del Estado que lo aleja de su tradicional rol omnipotente, para situarlo como un nodo más dentro de un ensamblaje de movimientos y estrategias que tienen lugar en un tiempo/espacio determinado. El autor sintetiza la idea sosteniendo que "trata de extraer las relaciones de poder de la institución, para analizarlas desde la perspectiva de las tecnologías, extraerlas de la función para retomarlas en un análisis estratégico y liberarlas del privilegio del objeto para resituarlas desde el punto de vista de la constitución de los campos, dominios y objetos de saber" (p. 82).

La categoría de gobierno que Foucault introduce a modo de profundización de sus análisis sobre el poder, abarca un campo que cruza desde las grandes estructuras de dominación hasta la nimiedad de las técnicas y procedimientos cotidianos que participan en la conducción de la conducta de los otros y la propia. El enfoque no dispone de una oposición jerárquica entre los elementos, sino que en un constante dialogo orienta su mirada crítica hacia los modos en que el sujeto es producido como efecto de un poder racionalizado.

El análisis gubernamental es actualizado por el grupo denominado anglofoucaultianos, quienes desarrollaron a partir de los años noventa una línea investigativa conocida como los governmentality studies, la cual aplica el marco conceptual de Foucault situado en la sociedad contemporánea neoliberal. Los análisis gubernamentales en este contexto, constituyen para los anglofoucaultianos un campo en el cual se reorganizan los problemas generales sobre el ejercicio del poder, inscribiéndose en su interior las temáticas de soberanía, disciplina y biopoder. Rose (1999) identifica como una especificidad de la estrategia moderna de gobierno la producción de regímenes de Verdad, el autor lo define como "un modo de análisis que da cuenta de la Verdad, quien tiene el poder de definir la Verdad, el rol de las diferentes autoridades y las condiciones epistemológicas institucionales y técnicas para la producción y circulación de Verdades" (p. 29).

Para comprender el modo en que circulan las relaciones que conectan la vida de los sujetos con las aspiraciones de las autoridades en las sociedades liberales avanzadas, los anglofoucaultianos articulan una metodología teórica a través del estudio de la interdependencia entre las Ilamadas racionalidades políticas y las tecnologías de gobierno. La primera es entendida por Miller y Rose (1992: 175) como política "campos discursivos de configuración cambiante en cuyo marco se produce una conceptualización del ejercicio del poder", los cuales pueden ser analizados tomando en consideración justificaciones morales de las formas de proceder de las autoridades, las nociones y objetivos de las política y las ideas sobre los roles que deben asumir cada autoridad, sean estas seculares, militares, religiosas, etc. 
La racionalidad política, en resumen, se erigirá como el marco de inteligibilidad a partir de cual se considerarán los valores que regirán el gobierno -justicia, libertad, ciudadanía, eficiencia económica y prosperidad entre otros- ; una epistemología sobre la naturaleza del objeto gobernado -sociedad, nación, población, economía- ; y, su articulación a partir de un idioma distintivo que funciona como maquinaria para la representación de la realidad pensable. Luego, las tecnologías de gobierno serán identificadas como "las estrategias técnicas y procederes a través de los cuales diferentes fuerzas buscan hacer operables programas y por medio de los cuales una multitud de conexiones son establecidas entre las aspiraciones de las autoridades y las actividades de los individuos y grupos" (MiIler y rose 1992:183). Estas nos remiten al enfoque microfísico del poder desarrollado por Foucault, cuestionándose a partir de aquellos mecanismos mínimos y cotidianos, que muchas veces se presentan como incuestionable, su funcionamiento como modelador de conductas.

Desde esta perspectiva, el análisis de las gubernamentalidades (de Marinis, 1999), se presenta como un despliegue de la extensa red que acopla racionalidades políticas y tecnologías de gobierno, sin darle primacía a ninguna instancia en particular. Se trata de identificar "por una parte, unas formas más o menos estructuradas y organizadas de pensamiento y saber, y unas prácticas, tácticas, técnicas, dispositivos, mecanismos orientados a modular la conducta" (p.17).

Cabe señalar que la gubernamentalidad no se presenta en estado puro, sino que en ella conviven racionalidades políticas decadentes con otras ascendentes, por lo que pueden compenetrarse técnicas avanzadas de gobierno con dispositivos de racionalidades anteriores.

La gubernamentalidad actual podría enmarcarse en la forma de gestión de la población característica de aquello que Deleuze y Guattari (1995) llaman Sociedad de Control, refiriéndose a una formación que a diferencia de la producción de subjetividad de las sociedades disciplinarias, no fijar identidades cerradas y delimitadas por los mu- ros de las instituciones, sino, que busca una modulación o control de elementos de la población que están constantemente variando. Este cambio es la expresión de un capitalismo que desplaza su centro asentado en la producción, hacia un patrón mercantil móvil, fluctuante que tal como afirma Deleuze (1995) "intenta vender son servicios, lo que quiere comprar son acciones. No es un capitalismo de producción sino de productos, es decir, de ventas o de mercados. Por eso es especialmente disperso." (p.153) La disciplina, como mecanismo de este poder, va progresivamente dejando atrás sus técnicas rígidas de sujeción para convertirse en inmanencia. Las diferentes instituciones que la conforman, se entretejen así, en una producción híbrida de subjetividad.

El funcionamiento de la economía dentro del sistema de ensamblaje biopolítico supranacional, tiene cabida a partir de la transformación de las fuerzas productivas, las cuales han redefinido por completo el trabajo y los lineamientos globales de la producción. Hardt y Negri (2005), apuntan a la informatización de la economía, como el motor de cambio en la naturaleza del trabajo, con esto refieren a la sistemática introducción de modelos de intercambio de información y conocimientos, caracterizados principalmente en el desplazamiento del sector productivo industrial, por el de producción de servicios. Los autores nombran este tipo de producción como trabajo inmaterial, en el cual ya no requerida una fuerza corporal única convocada por la labor industrial y respaldada por las técnicas disciplinarias, sino que necesita de las habilidades analíticas, simbólicas, creativas y emocionales, es decir, la vida en su conjunto.

La forma de gobierno que se enmarca en este contexto inserta estrategias novedosas para conducir el medio social. El neoliberalismo como expresión gubernamental tiene entre sus principales transformaciones la modificación de la relación entre sujetos/política/expertos. La conducción de conducta en la nueva configuración Ilama al sujeto a realizarse a sí mismo y a maximizar su calidad de vida mediante su propia elección entre servicios a los cuales opta ya no como ciudadano 
sino como consumidor -consumidor de servicios de salud, de educación, de formación-.

La nueva forma de relacionarse con la autoridad consiste en que los expertos dotan a los sujetos de saberes o marcos que unifican un vocabulario, juicios y explicaciones. Una vez que estos marcos son interiorizados los sujetos adquieren una autonomía regulada respecto a sus vidas, la cual orientan hacia el horizonte de la realización personal y la felicidad como parte de un proceso individual. En relación a los nuevos sistemas productivos inaugurados en la Sociedad de Control, la gubernamentalidad neoliberal instala una forma de relación social guiada por el modelo "empresa". Esto se entiende considerando la transformación de los sistemas económicos, donde el modelo de producción económico cerrado o burocrático típico del fordismo pierde eficacia frente a los modelos productivos globales y flexibles que se constituyen ya no solo en el espacio físico de la fábrica sino que toman como marco de intervención la sociedad en su conjunto.

La "empresa" se sitúa en este marco, como un modo de conducción de la conducta no solo a nivel organizacional, la cual se generalizó hacia todos los individuos, aludiendo a "características como la iniciativa, la aceptación de los riesgos, la confianza en sí mismo, y la capacidad de admitir responsabilidad por uno mismo de sus actos" (Keat, 1990 citado por Du gay 2003: 256). Inaugurando de esta forma un modo de subjetividad que Du gay (2003) llama empresario de sí mismo, apuntando con esto al sujeto "cuya autonomía se juega en su capacidad para ser perpetuamente receptivo a y modulado por las incesantes modificaciones del ambiente" (p. 260).

\section{Producción de subjetividad en discapacidad}

Podemos situar el momento en que la discapacidad es paulatinamente considerada como objeto del cual producir una categorización y un conocimiento especifico, en torno un proceso ini- ciado en el S.XVIII, que Foucault Ilama aumento de los efectos de poder. Caracterizado por una inclusión analítica de los elementos antes marginados a través de la formación de un saber sobre ellos, que posibilita el control de la población a través de los aparatos gubernamentales. El autor lo define como la formación de un poder ligado a "toda una serie de mecanismos que aseguran la formación, la inversión, la acumulación, el crecimiento del saber" (Foucault, 2000: 55)

En relación a este saber se van constituyendo diversas disciplinas que a partir de marcos nosológicos, de clasificación y registro capturan la legitimidad de hablar sobre ellos. En el caso de la discapacidad quienes constituyeron este saber fueron por un lado el discurso médico a partir de su hegemonía sobre el saber anatomo-fisiológico de los cuerpos (Le Breton, 2002), y por otro, la psiquiatría que se organizó como disciplina a partir de la producción de saber en torno al anormal (Foucault, 2000)

Ambas disciplinas amparadas en su estatus científico formulan discursos con efectos de verdad y poder, y se adjudican el monopolio de la legitimidad: "no hay producción de conocimiento o práctica científica, incluyendo a la medicina que sea independiente del poder" (Lemm, 2010: 88). En efecto, se estructuran formas de poder/saber, es decir, conocimientos apoyados en un poder, que luego justificarán las prácticas de intervención sobre sus objetos. La construcción de personas con discapacidad dependerá de estos sistemas de clasificación y su producción de efectos de poder.

En el caso de la discapacidad, el avanzado saber que se desarrolla a partir de los años ochenta, permite la profundización de prácticas de intervención sobre estos cuerpos desplegando un "amplio rango de políticas públicas de salud, intervención en la higiene de la población y muy importante, formas de clasificación, definición y control de la población" (Lemm, 2010: 88) propios de la gestión biopolítica.

La importancia de la biopolítica en este sentido, es que a través de la rehabilitación pone en funcionamiento un dispositivo que conjuga una 
serie de discursos, prótesis, sistemas educativos etc. que funcionan como tecnologías, que en tanto actos productivos "dan lugar y visibilidad a las discapacidades" (Ortuzar, 2009:71).

El dispositivo rehabilitador constituye una serie de materialidades o niveles donde el cuerpo es intelegido y configurado en relación a la biología, la anatomía, la fisiología, la vida, etc. (Butler, 2002). Respecto a los cuerpos que no entran en la hegemonía de lo normal, esta materialización se encarna en la medida que todas esas disciplinas se forman como tecnologías de corrección del cuerpo discapacitado, funcionando como principio normalizador. La biopolítica modula los cuerpos a partir de este principio, el cual funciona estableciendo la lejanía o cercanía de la norma a partir de la clasificación y la corrección: "su función no es excluir, rechazar, al contrario, siempre está ligada a una técnica positiva de intervención y transformación, a una especie de proyecto normativo" (Foucault, 2000: 57).

La rehabilitación y las políticas de intervención del cuerpo discapacitado designan a partir de estas disciplinas que las legitiman, una desviación respecto al cuerpo legítimo: "a partir de la incorporación en los esquemas de percepción, sentimiento y acción de la discapacidad como una oposición a la capacidad, como cuerpo enfermo inútil por oposición al cuerpo sano útil (como "enfermedad" -mala- por oposición a salud -buena-), se adquiere antes de todo orden consiente una valoración negativa de la discapacidad" (Ferrante \& Ferreira, 2007:8). En relación a la norma, Butler (2002) enfatiza que la materia de los cuerpos como efecto de una dinámica de poder es "indisociable de las normas reguladoras que gobiernan su materialización y la significación de aquellos efectos materiales" (p.60)

El proceso a través del cual los cuerpos se materializan adoptando cierta norma corporal se entiende a partir de la conformación de los sujetos, lo cual, mediada por el poder va incorporando registros normativos. Judith Butler (2002) señala que la materialidad es un efecto disimulado del poder, que tiende a naturalizarse a partir de la constitución y la formación de un campo epistémico y un conjunto de conocedores que "se dan por sentados como fundamentos pre-discursivos" (p.64). Así, el principio normalizador se establece como principio cultural del cuerpo, estableciendo aquellas partes del cuerpo o aquellas corporalidades que deben ser excluidas. En efecto, cuerpo y subjetividad no funcionan como dos apartados distintos, sino que forman un continuo, una dinámica donde uno remite al otro. La materialidad del cuerpo contiene en su formación una modulación subjetiva, situada en un proceso histórico determinado que se va plegando para dibujar un contorno siempre fluido. Al respecto, Landa y Marengo (2010) señalan que "cada constelación cultural se define en parte por el entramado de ficciones narrativas que diagraman la plataforma simbólica e imaginaria a partir de la cual se constituye, un mismo desplazamiento, aquello que Ilamamos 'cuerpo' y aquello que denominamos 'sujeto'" (p.170)

El cuerpo, sin embargo, no solo es el registro material de una cierta producción de subjetividad, sino que en la dinámica misma de conformación, ambos se van reflejando como parte de una relación mutable de múltiples efectos/causas. Lemm (2011) acerca de la relación dinámica entre materialidad del cuerpo y la subjetividad, asevera que esta no tiene predeterminado un sujeto o una mera configuración discursiva "sino la articulación de una serie de dispositivos entendidos como una serie de prácticas y discursos, de saberes y de ejercicios, dirigidos a crear cuerpos dóciles, pero a su vez cuerpos libres" (p.61).

Los regímenes de verdad que intervendrán en el cuerpo discapacitado, ya sea simplemente clasificándolo como tal, o aun mas, directamente sometiéndolo al dispositivo rehabilitador, no solo estarán modificando el cuerpo y su organización, sino que sincrónicamente se estarán inscribiendo en la subjetividad misma de aquella corporalidad.

En el marco de la gubernamentalidad las dinámicas de inclusión/exclusión social se entienden como parte de procesos de gestión y de modulación de subjetividades. En este sentido, si bien 
el control social como ejercicio positivo del poder busca la inclusión de toda la población, actualmente para obtener mayor efectividad ante la producción y proliferación de subjetividades híbridas, se vuelve preciso establecer estrategias de gerenciamiento más focalizadas, las cuales dan cuenta de formas de exclusión que operan sobre campos más específicos de control.

En el caso de la discapacidad, estos cuerpos constituidos a partir de su exclusión de las fronteras de la norma y señalados a partir de un estigma (Goffman, 2006) son integrados en términos gubernamentales, sea esto, generando todo un marco específico que permite su intervención social: cierto saber legítimo, un marco legal y la articulación de políticas públicas, entre otros. Sin embargo, estos dispositivos de intervención no totalizan la producción de subjetividad de los cuerpos con discapacidad, debido a que también son cruzados por otros dispositivos de intervención, los cuales paradójicamente funcionan en parte gracias a la exclusión de éstas las corporalidades 'anormales'. En efecto, las variaciones que tienen las normas fluctuantes de la Sociedad de Control generan, a partir de procesos de desarrollo de conocimientos o del requerimiento productivo -entre otros-, nuevas formas de exclusión cada vez más complejas, de las que se hace difícil dar cuenta a cabalidad.

Para conocer los procesos de exclusión/integración del cual son parte los cuerpos con discapacidad, es necesario comprenderlos como articulaciones simultáneas, que tienen su origen principalmente en la forma de gestión de la gubernamentalidad neoliberal. La cual se caracteriza entre otras cosas por la importancia del factor mercantil presente en las diferentes prácticas de gobierno de los cuerpos, infiltrándose a través de los dispositivos que constituyen la materialidad de éstos en el sentido planteado por Butler.

La construcción corporal de la discapacidad que naturaliza y media la relación con el sujeto se visibiliza a partir del saber sobre la discapacidad que es difundido principalmente por instituciones como la ONU y la Organización Mundial de la Salud quienes trazan el lenguaje, el conocimiento y las prácticas derivadas desde el enfoque asistencial propio de la medicina hacia el enfoque social en cual intervienen otras instancias.

Los efectos de poder que se evidencian a partir de esta visibilización en relación al paso de la gubernamentalidad anterior a la neoliberal distinguen entre la producción de dos tipos de subjetividad. La primera se despliega desde las primeras consideraciones institucionales del cuerpo discapacitado cuya orientación está dada por la publicación de la OMS en el año 1980 de la Clasificación internacional de deficiencias, Discapacidades y Minusvalías. Manual de Clasificación de las consecuencias de la Enfermedad (CIDDM). Este informe presenta la triple tipología de deficiencia en relación a la anormalidad psicológica, fisiológica o anatómica; discapacidad referida a la ausencia de la capacidad de realizar una actividad que se considera normal; y minusvalía entendida como la situación desventajosa a consecuencia de una deficiencia o discapacidad (Casado, 2001). Brogna (2009), señala que este marco conceptual común elaborado desde la ciencia "confirma la individualidad del déficit y el determinismo orgánico de lo patológico, lo anticipa, lo diagnostica, lo nomina, le pone apellido, lo clasifica y ofrece un abanico de propuestas para su reparación" (p. 220)

La perspectiva de la CIDDM, situada en el modelo médico de entender la discapacidad, es nombrada por Barnes (2009) como tradicional individualista, y supone la adaptación del cuerpo al medio social y no al revés., hasta aproximadamente la década del ochenta, y establece la producción de subjetividad del discapacitado ligada a la fijación del cuerpo a una norma estable, que lo determina como desvalido, paciente y dependiente del accionar asistencialista.

La segunda línea de producción subjetiva se moviliza a partir del cambio que presenta la OMS en el año 2001 con la publicación de una nueva clasificación Ilamada Clasificación internacional del funcionamiento de la Discapacidad y la Salud (CIF). La CIF fue el documento que recoge lo que para algunos autores es el nuevo paradigma de 
entender la discapacidad, promovido por la OMS. Conjuga el modelo medico de la CIDDM con la mencionada interpretación sociopolítica, articulando un modelo bio-psico-social (Egea, Sarabia, 2001) que intenta superar la perspectiva bio-médica de entender la discapacidad, el cual sin dejar de lado el factor médico, pone mayor énfasis en factores contextuales que impiden el normal desarrollo de los individuos.

En esta nueva definición, la discapacidad, será el término genérico que engloba los anteriores conceptos de deficiencia y minusvalía, en un esquema triple que toma como eje las funciones en positivo del cuerpo, la actividad y la participación. Esta sería la principal diferencia con su antecesora, no relacionar a la discapacidad directamente a la enfermedad sino a la salud y sus estados relacionados.

Sin embargo, aun cuando se ha planteado que la CIF refleja el cambio de paradigma del nuevo modelo social, esta aun sigue anclada en el individuo como "punto de partida para el análisis de las 'funciones y actividades corporales" (p. 106), donde la participación y el entorno continúan estancadas en las circunstancias personales. Convirtiéndose en un intento débil de enfatizar en los factores contextuales: aun cuando están presentes no entrega las herramientas adecuadas para medirlos, o para evaluar siquiera las tendencias 'discapacitadoras' de los programas y prácticas públicas (Barnes, 2009).

\section{Marco gubernamental de inteligibilidad de la figura del discapacitado en Chile}

El ejercicio de la gubernamentalidad en Chile que visibiliza a la discapacidad fue dividido en dos momentos significativos para el quehacer normativo y político. El primero comprende desde la promulgación de la primera ley 19.284 en el año 1994 hasta la publicación en 1999 de la Política Nacional para la Integración Social de las personas con discapacidad. Y el segundo período se corresponde con el Plan Nacional de Acción para la Integración Social de las Personas con Discapacidad 2004-2010, que va desde Primer Estudio Nacional de la Discapacidad, en el año 2004, a la promulgación de la Ley 20.422 que establece las Normas sobre Igualdad de Oportunidades e Inclusión Social de las Personas con Discapacidad, en el año 2010.

El análisis de las categorías identificatorias en cada período fue organizado a partir de ejes correlaciónales relativos al enfoque gubernamental. Si bien, a efectos del presente artículo no es posible presentar detalladamente los hallazgos que se dieron a partir de esta genealogía de los discursos representativos de cada período, a continuación se exponen a modo de resumen algunas líneas concluyentes que dan cuenta de determinado proceso de producción subjetiva.

\subsection{Políticas de intervención sobre el cuerpo discapacitado}

Las últimas dos décadas de consolidación y profundización del modelo neoliberal, que han marcado la postdictadura chilena, han implicado una serie de transformaciones en los modos en que el Estado articula las prácticas de gobierno, desarrollándose tecnologías que apuntan a un control biopolítico cada vez más dinámico, y aún así capaz de actuar de manera minuciosa y contingente. Esto se comprende a partir del desplazamiento del eje de acción del aparato estatal hacia un rol que, si bien no deja de lado el gobierno de la población, lo hace en tanto mediador entre esferas consideradas como no-políticas, como el ámbito económico, el cual se abre paso en la prestación de servicios que antes eran netamente parte de la administración pública. Así, se han establecido por ejemplo relaciones no directas de la ciudadanía con los servicios estatales de asistencia, los cuales ven reducida su posición cada vez más a la de expertos que se mueven entre las esferas privados.

La actual Ley 20.422 es enfática en varios artículos al respecto, señalando por ejemplo: 
Esta ley, además, promueve la cooperación público-privada, entendiendo que la discapacidad es un tema que afecta a toda la sociedad. Es, por lo tanto, deber de todos contribuir a superar las barreras que impiden o dificultan la plena integración de las personas con discapacidad. (Historia de la Ley 20.422: 905)

A pesar de este desplazamiento de la acción estatal nos encontramos con una intención de protección social que deviene una vez avanzado el proceso de neoliberalización. Esto que podría leerse como una contradicción con los lineamientos antes mencionados de este modelo, lejos de significar una vuelta a un modo de gobierno basado en el Estado de bienestar, las políticas derivadas de la protección social se articulan como una estrategia que supone el mantenimiento del equilibrio macroeconómico del modelo de desarrollo neoliberal; es decir, el mercado continúa siendo la principal forma de organización económica y el Estado un mero regulador, pero se complementa con políticas de protección a grupos vulnerables en base a políticas focalizadas. La falla sistémica que anuncia que el crecimiento económico en Chile reproduce las condiciones de desigualdad en su población se mantiene (Camargo, 2007), pero se pretende garantizar el bienestar social entregando herramientas a los sectores más pobres, se busca "maximizar las oportunidades para que los individuos alcancen su bienestar" (Waissbluth e Inostroza, 2007: 286). En este marco la nueva ley 20.422 se sitúa dentro de una estrategia general de gobierno por parte del Estado. Esto se expresa en la siguiente cita extraída del discurso de promulgación de la ley:

(...) los derechos de las personas con discapacidad se han convertido en un componente fundamental en la construcción del sistema de protección social comprometido por la presidenta Bachelet al 2010 (Historia de la ley 20.422: 351)

La emergencia del aparato estatal como eje mediador y de gestión, se remite a fijar temporal e institucionalmente las tecnologías de gobierno necesarias para la producción de los sujetos requerido por estas formaciones gubernamentales, cuidando de controlar los desbordes que se producen. De esta manera, son establecidas estrategias legitimadas a partir del régimen de verdad generado en y por el saber difundido sobre los sujetos de gobierno, junto a los juegos de poder entramados en los dispositivos que forman nexos de identificación que unen a estos sujetos con los anhelos de la gubernamentalidad. Esto crea campos de inteligibilidad sobre las mentalidades y los cuerpos, lo que permite hacer aparecer ciertas vidas como posibles en términos de la racionalidad política.

Siguiendo esto, a partir del análisis de las categorías identificatorias se observó que en los dos períodos destacados es posible reconocer las continuidades y rupturas que emergen en dichos campos de inteligibilidad.

El primer período, correspondiente a los años 1992-1999, se desarrolla bajo el interés de gobierno de fortalecer el marco democrático post-dictatorial. La discursividad del entonces presidente Aylwin da cuenta de la articulación de una racionalidad política de corte democrático-liberal, cuyos fines de gobierno se regirán por un marco institucional integrador, y por prácticas que aseguren el desarrollo de una economía de mercado abierta al exterior. En este escenario, el Estado asume un rol que por un lado es garante y promotor de políticas tendientes a la integración controlada de la población, y por otro de regulador y fomentador del sistema económico. Las tecnologías requieren establecer una identificación con los sujetos Ilamados a hacerse parte, donde estar considerados por el marco institucional, y por la democracia se vuelva un anhelo de ellos mismos, así como el sistema económico se vuelva motivo de consenso social.

Dentro del marco de una economía de mercado abierta hacia el exterior, en el que la iniciativa privada y el dinamismo empresarial juegan un rol preponderante, y el Estado cumple principalmente funciones de regulación y fomento sobre la base de reglas claras y estables, polí- 
tica que concita generalizado consenso nacional, el país ha experimentado en el último año importantes avances. (Aylwin, 1992)

Por otra parte, el segundo período, que considera los años 2004-2011, se corresponde a una expansión de los marcos democráticos-institucionales como correlato de la consolidación y apertura del modelo económico chileno hacia los mercados internacionales.

Los cálculos de gobierno se encontraban enfocados en la continuación del proceso de modernización estatal y la articulación de sus instituciones para producir mayor eficacia, tanto en el control de las transformaciones sociales, como en el fomento del crecimiento económico.

En este escenario los esfuerzos se centran en asegurar un buen posicionamiento del país en los mercados globales y una revisión de los mecanismos políticos y legislativos internos para adecuarlos a las recomendaciones de los organismos internacionales.

Respecto a estos mecanismos, la necesidad de una nueva normativa para las personas con discapacidad surge a partir del:

(...) actual debate que se lleva a cabo en la Organización de las Naciones Unidas para la elaboración de una Convención Internacional Comprensiva e Integral sobre la Protección y Promoción de los Derechos y Dignidad de las Personas con Discapacidad, iniciativa apoyada por nuestro país desde sus inicios, nos plantea nuevos desafíos que demandan la adecuación de nuestra legislación interna (Historia de la Ley 20.422: 6)

Sumado a esto, los datos del primer Estudio Nacional de la Discapacidad (ENDISC) señalan a la discapacidad como un fenómeno importante en cuantía dentro del país, 12,9 \% de total de la población presenta alguna discapacidad. Queda evidenciada una relación directa con la pobreza consecuencia de la reducida eficacia las políticas efectuadas para superar la situación de vulnerabilidad de este grupo social, y la baja participación de las personas con discapacidad dentro de la actividad económica $(29,2 \%$ del total de las personas con discapacidad en edad de trabajar efectivamente lo hace).

Durante este período se genera una identificación ya no directamente con la democracia como ideal o anhelo de los ciudadanos, sino una identificación relativa al discurso de la diversidad como factor necesario para el progreso y desarrollo del país, y del anhelo de mejorar las condiciones de la calidad de vida y el acceso a las oportunidades.

La apelación constante al concepto Diversidad comienza a surgir en el período trazado entre los años 2004 y 2011 y su importancia se puede constatar en el discurso de promulgación de la Ley 20.422:

Con esta ley, estamos haciendo realidad aquello que siempre hemos creído: que una patria íntegra es la que cuenta con todos sus ciudadanos, la que no olvida a nadie, la que hace de la diferencia y la diversidad una riqueza, la que acoge a todos sus hijos en el desarrollo, la justicia y la realización personal. (Historia de la Ley 20.422: 906)

La Diversidad como valor a celebrar conforma un sustento tanto democrático como moral, es señalada como correlato de la inclusión y elemento necesario para el progreso y desarrollo del país.

Característica de las democracias liberales, la inclusión de la diversidad, aún cuando en el discurso alude al respeto de la diferencia, finalmente actúa como factor de control de ésta, haciéndola asimilarse a un "nosotros" legible y regulable en términos de poder (Duschatzky y Skliar, 2000). La inclusión de la diversidad en la práctica se realizará en la medida que sea atravesada por las políticas que la enuncian y que sea capaz de hacer suyas las oportunidades que son ofrecidas por el neoliberalismo y que el Estado le ayudará en cierta medida a acceder.

En este segundo período, el ciudadano es interpelado a maximizar su calidad de vida, a través del ejercicio de su oportunidad de elección responsable entre los diversos servicios y productos ofertados. 
El segundo operador discursivo destacado es la calidad de vida. Esta es definida como una "combinación de las condiciones de vida y la satisfacción personal ponderada por una escala de importancia que refleja los valores personales, las aspiraciones y las expectativas de la persona" (Jiménez, 2006: 74)

El Estado anuncia su cambio de eje desde una acción asistencialista al de proveedor de calidad de Vida, señalando en el Artículo $4^{\circ}$ el objeto de sus programas respecto a la discapacidad:

Es deber del Estado promover la igualdad de oportunidades de las personas con discapacidad. Los programas destinados a las personas con discapacidad que ejecute el Estado, deberán tener como objetivo mejorar su calidad de vida, principalmente, a través de acciones de fortalecimiento o promoción de las relaciones interpersonales, su desarrollo personal, la autodeterminación, la inclusión social y el ejercicio de sus derechos. (Ley 20.422, 2010)

La calidad de vida se torna tanto categoría democrática-institucional como categoría moral de identificación, al apelar a la inclusión social y ejercicio de sus derechos, al mismo tiempo que al desarrollo personal y la autodeterminación como aquello que se debe considerar para lograr el bienestar individual

En ambos períodos se produce la intervención del cuerpo de la persona discapacitada convirtiéndolo en el lugar de aplicación de políticas tecno-médicas de transformación y control.

Durante el primer período nos encontramos con incipientes definiciones de prevención y rehabilitación basados en el mejoramiento del funcionamiento corporal, durante el segundo período estos ya se han especializado articulándose como tecnologías biopolíticas totales sobre los cuerpos y los procesos de normalización haciendo más eficiente su inserción en el ámbito productivo. Esto queda en evidencia a partir de las modificaciones que se presentan en ambos períodos del sobre las definiciones de discapacidad y los consecuentes modos de rehabilitación, y en aquellos aspectos que se mantienen intactos como el certificado de discapacidad.

La conceptualización de discapacidad es el primer instrumento de control que despliega a partir del saber sobre los sujetos, la cual servirá para operacionalizar la condición de quienes serán beneficiarios de esta ley.

En la ley 19.285 es el correlato de los marcos de inteligibilidad sobre discapacidad difundidos por organizaciones internacionales como las $\mathrm{Na}-$ ciones Unidas, desde la cual se apela no solo al cuerpo y su déficit como fenómeno individual sino que también éste en relación con el entorno social. Según esto una persona con discapacidad será:

(...) aquéllas que tienen una restricción o ausencia de la capacidad de realizar una actividad en la forma o dentro del margen que se considera normal para ser humano y cuya causa es una "deficiencia", esto es una pérdida o anormalidad de una estructura o función sicológica, fisiológica o anatómica (Discurso presidencial, Historia de la Ley, 1992)

En esta definición existe una clara referencia al ajuste de los cuerpos hacia una norma, si bien no es la definición textual que finalmente primará en la Ley, da cuenta del marco en el cual la ley posteriormente rearticulará esta definición señalando ya no el margen que se considera normal para el ser humano sino el hecho de que "vea obstaculizada, en a lo menos un tercio, su capacidad educativa, laboral o de integración social" (Ley 19.289). La rehabilitación que se propone en función de esta definición localiza en la recuperación de la funcionalidad del cuerpo los aspectos necesarios para la integración social, educativa y laboral.

Las trasformaciones que incluye la ley 20.244 en sintonía con el modelo bio-social de entender la discapacidad, articulan una definición enfoca desde una perspectiva de derechos, determinando una definición de discapacidad acorde a la realizada por la Organización Mundial de la Salud a través de la CIF, alejándose de la concepción me- 
ramente tecno-médica que imperaba, apuntando al entorno y las barreras que produce como los agentes sustanciales del problema de la discapacidad. La discapacidad queda definida del siguiente modo:

Persona con discapacidad es aquella que teniendo una o más deficiencias físicas, mentales, sea por causa psíquica o intelectual, o sensoriales, de carácter temporal o permanente, al interactuar con diversas barreras presentes en el entorno, ve impedida o restringida su participación plena y efectiva en la sociedad, en igualdad de condiciones con las demás. (Ley 20.422, Artículo 5, 2010)

La referencia al cuerpo y su déficit se desplaza en importancia para apuntar a su participación en la sociedad, y los obstáculos que impiden ese acceso. En esta definición se hacen parte las categorías tecno-médica y democrática-institucional, fijando una mirada más amplia del sujeto, donde su integración queda determinada por dos ejes, la superación personal de sus deficiencias y la reorganización del entorno para su accesibilidad.

La participación de las personas con discapacidad es fomentada a partir tanto de la transformación del entorno como la del sujeto, cuyo movimiento debe apuntar al principio definido por la ley como vida independiente.

La rehabilitación a partir de este enfoque es vista como integral y comunitaria. La característica de integralidad hace referencia a las diferentes dimensiones que puede convocar el dispositivo. La base comunitaria de la rehabilitación hace alusión a la responsabilidad que debe asumir la sociedad o el grupo social próximo a la persona con discapacidad para facilitar su participación según los parámetros que la misma sociedad establece.

Tanto las definiciones como los procesos de rehabilitación tienen alcances y efectos directos a partir del certificado de discapacidad creado por la ley 19.284 y continuado por la posterior normativa. Este se articula como una tecnología netamente disciplinaria, desde la cual es posible separar al cuerpo en diferentes órganos, partes, sensibilidades, con tal de identificar aquellas que no se ajustan a la norma o las relaciones posibles entre ellas para definir una cierta habilidad. Se compone una nueva serie de saber/poder que registra al cuerpo según su posibilidad de corrección o la necesidad de potenciar otra parte en la búsqueda del horizonte trazado como funcionalidad.

La importancia que reviste este procedimiento que se fija como obligación para acceder a los beneficios de la ley, es decir como requisito de integración, da cuenta del funcionamiento de una norma que hará de estos cuerpos un sujeto válido en los términos de la gubernamentalidad imperante. Aquel sujeto que desea integrarse deberá aceptar ser definido y caracterizado en los términos elaborados por la COMPIN. Deberá aceptar el rango sobre el cual es medido.

Si bien en ambos períodos este control biopolítico no presenta modificaciones en términos legales de gestión o administración, el cambio se da en función del contexto y el enfoque diferenciado de las leyes.

Esto se puede observar en la definición de discapacidad inscrita en cada período donde en el primero podemos ver un énfasis en la deficiencia o pérdida o anormalidad de una estructura o función psíquica, fisiológica, o anatómica que trae vinculado un proceso de rehabilitación que busca la prestación de servicios oportunos para la recuperación de la funcionalidad y el mantenimiento de aquella estructura o función corporal. Mientras en el segundo período la discapacidad es definida relacionando el cuerpo y su parte en déficit con el entorno lo cual conlleva a un proceso de rehabilitación integral que busca proporcionar o restablecer funciones, compensar la pérdida o falta de una función o limitación funcional y el desarrollo de conductas, actitudes y destrezas que permitan la inclusión según la necesidad de interacción con el entorno, se hace referencia en especial al sector educativo y laboral.

En el primer período el dispositivo rehabilitación se articula en torno a una norma hacia la cual hace encajar el cuerpo según la información 
recibida por la previa categorización y clasificación realizada por la COMPIN. El cuerpo definido como discapacitado es sometido a diferentes procesos normativos que buscan corregir la disfuncionalidad identificada. Se accede al constructo tecno-médico de conocimiento que pauteará al cuerpo discapacitado sometido a rehabilitación, se le dotará de las prótesis necesarias y se modificará el cuerpo a partir de esto. Además el sujeto será intervenido a nivel de su mente y psiquis a través de un tratamiento psicológico que actuará a partir de la información sobre su personalidad obtenida gracias al certificado de discapacidad, dotándolo de un conocimiento experto sobre sí mismo.

En el segundo período caracterizado por una comprensión de la discapacidad a partir del medio social, el dispositivo rehabilitación se articulará de modo más complejo para configurar no la funcionalidad de determinado órgano, o parte del cuerpo, sino para producir una novedosa organización de éste en conexión con agente externos a él como las prótesis, las ayudas técnicas y principalmente lo que la ley 20.422 Ilama comunidad o entorno social. En este sentido la información recopilada por la COMPIN a partir del ejercicio netamente disciplinario se utilizará pero ya no para ajustar al cuerpo a la norma rígida de lo predeterminadamente deseable, sino no para definir una modulación de las posibles habilidades y capacidades del sujeto en relación con normas móviles propias de los requerimientos diversificados y flexibles de la Sociedad de Control.

La intervención multidimensional del cuerpo que se define a partir de la ley 20.244 en pos del principio de autonomía de los sujetos lo hará capaz de responder a las demandas complejas y llevar a cabo tareas diversas en forma adecuada. Esto en cuanto a lo que se hace referencia constantemente es a las habilidades definiendo con esto no solo una determinada funcionalidad sino un conjunto de conocimientos, motivaciones, valores éticos, actitudes, emociones, y otros componentes sociales y de comportamiento que se movilizan para lograr al sujeto de la inclusión capaz de optar a las oportunidades del medio social y garantizar de este modo su calidad de vida. Ahora este sujeto integrado deberá responder a la posibilidad de validez que se le dio a partir de esta gestión de su cuerpo a través de su participación activa en el medio social.

El efecto último de estas tecnologías aplicadas sobre los cuerpos aún cuando estos accedan o no, o cumplan o no con la finalidad de los dispositivos, es la producción de un régimen de verdad, de un campo de inteligibilidad sobre la discapacidad desde donde se interpelará a los sujetos y se les dotará de un cierto lenguaje sobre ellos mismos, de les adjudicará la necesidad de la intervención de estos dispositivos, generando a la discapacidad y al sujeto interpelado por este fenómeno como relato de la integración o la inclusión según sea el caso.

\section{A modo de conclusión: Subjetividades producidas en torno a la discapacidad en Chile}

Como ha quedado estipulado los procesos de identificación enunciados a través de las formas de designación de la discapacidad contenidos en los documentos gubernamentales son productoras de subjetividad, sin embargo, hay que tener presente aquello en tanto que documentos se tensionan a partir de su enfrentamiento con situaciones de facto. En efecto, los programas elaborados a partir de los lineamientos de las normativas y las políticas estatales se encuentran en la práctica con formas de acción y actuación que muchas veces los contradicen o se desvían de su eje referencial. Estas dos esferas, aquello enunciado en los documentos y lo que efectivamente sucede, conllevan tensiones en donde las instituciones y las subjetividades se encuentran y se producen. De aquí que se hace necesario por un lado identificar las prácticas y saberes que instituyen los documentos como leyes, programas políticos, convenciones, etc., en tanto hacen inteligible, pensable y actuable cierta subjetividad en un determinado contex- 
to, y por otro lado tensionar esta subjetividad a partir de los quiebres que se provocan enfrentándolo con aquello que efectivamente sucede.

En el caso de la discapacidad, el campo institucional articula una serie de dispositivos que realizan una administración técnica de la diferencia corporal impulsada por el discurso de la integración en los años noventa y luego de la inclusión a partir de los años dos mil. En este sentido, las transformaciones que en diferentes contexto argumentativos se han dado a partir de la primera normativa chilena sobre discapacidad en el año 1994, se han dirigido a volver gobernable a aquellos sujetos cuyas corporalidades no responden a los criterios de normalidad vigentes disminuyendo los riesgos de un desborde a partir del control de esta población.

A partir de lo anterior se puede argumentar que el progreso de la gubernamentalidad neoliberal en Chile ha desplegado dos tipos de estrategias que Grinberg (2008) señala como propias del problema del control en los últimos años "aquellas vinculadas a la inclusión y aquellas que aceptando la inexorable de la marginalidad procuran neutralizar los riesgos/peligros" (p. 262).

Los documentos referidos a la discapacidad analizados dan cuenta del primer tipo de estrategias, desde las cuales se planifica el hacer vivir de la biopolítica, articulando un conjunto de tecnologías de integración/inclusión que apuntando a la rehabilitación remiten finalmente a la búsqueda y construcción de una moral ligada a la vida independiente, la autonomía y a la reorganización del cuerpo de acuerdo a aquello posible de potenciar para la producción.

Aquel sujeto posible de la inclusión se producirá en el marco de los discursos gubernamentales que determinan a través de sus tecnologías integradoras y del constructo Saber, la inteligibilidad y visibilización de la discapacidad, produciendo un saber sobre sí mismo que actúa como núcleo biopolítico que produce cuerpos dóciles al servicio de la producción del capital.

Desde este marco es posible hablar de la producción de una subjetividad empresario de sí mis- mo, que si bien en el caso de la discapacidad estará mediada por un aparataje institucional tanto público como privado, apunta a la constitución de una autonomía efectiva y responsable como soporte moral de la productividad (Martínez, 2010) propia de la interacción de liberalismo y democracia de la gubernamentalidad que se forja en Chile. Este proceso se articula a partir del cual los objetivos de las autoridades políticas, sociales y económicas se emplazan "en el interior de las elecciones y compromisos de los individuos situándolos en redes reales o virtuales de identificación a través de las cuales pueden ser gobernados" (Rose, 1997: 18)

La formación moral y la promoción de los valores de vida independiente y la autonomía como correlato del autogobierno de los sujetos apunta a crear sujetos que busquen maximizarse, buscar su felicidad y calidad de vida asumiendo aquel yo previamente narrado por el discurso elaborado desde diferentes disciplinas como la psicológica, en este sentido Grinberg (2008) señala que "dotar al yo de una identidad coherente constituye el eje de una nueva programática política, de una ética que se estructura en torno al proyecto de vida $y$ del hacer yo" (p. 198).

Tanto el sujeto integrado de la primera etapa (1994-1999) como el sujeto incluido de la segunda (2004-2011) son el resultado de la intervención de esta programática política y sus tecnologías, como lo son un proceso de rehabilitación exitoso, el acceso a las ayudas técnicas necesarias y la propia experiencia vital narrada desde un determinado paradigma. Puesto que la autonomía y la vida independiente son atributos impensables sin una inserción eficaz en el sistema productivo. Es por esto que el proceso de modificación corporal, en pos de reparar o compensar la insuficiencia, se vuelve imprescindible. Sin embargo este proceso de modificación no se experimenta como una manifestación rígida de saberes o normas a la cuales ajustar el cuerpo, sino que lleva a cabo análisis dinámicos de las potencias y habilidades a desarrollar según las características de cada sujeto. 
La programática legal y política fomenta la asunción de una corporalidad que acorde a los modelos productivos, sea flexible, capaz de adaptación, consciente de sus potencialidades y limitaciones. En este punto toma sentido la inclusión posible de aquellos cuerpos que si bien no pueden ser productivos en términos de la fuerza o capacidad corporal clásica de las labores industriales, si pueden inscribirse de acuerdo a sus habilidades creativas o afectivas en las dinámicas productivas llamadas inmateriales (Virno, 2002).

El relato de la inclusión interpela al sujeto a partir de la producción subjetiva que da lugar al empresario de sí mismo, este es quién en la gubernamentalidad neoliberal tiene un lugar en base a sus habilidades desarrolladas como potencial de producción, y al autodisciplinamiento como resultado de una moral basada en los valores de vida independiente y autonomía como soportes de la calidad de vida. Entendiendo que la calidad de vida es posible en tanto se accede a las oportunidades que acorde al nuevo rol estatal pueda ofertar el mercado, la inclusión estará íntimamente ligada a las gestiones que el sujeto realice para maximizar su vida, modelando y gobernando sus capacidades y competencias. El sujeto empresario de sí mismo necesitará de la autodisciplina que le permitirá el acceso a la calidad de vida.

En este plano de hechos aquel sujeto que no pueda acceder a la inclusión, que no pueda hacer un empresario de sí mismo, ya sea por lo privativo que resultan los servicios estatales, o por la situación de marginación que aqueja a la mayoría de la población considerada discapacitada, queda a merced de la precarización de su condición, convirtiéndose en vida que no merece ser vivida.

Se define así la tensión fundamental que se establece con los marcos programáticos de inclusión para personas con discapacidad contenidos en leyes y políticas de acción estatal, conflicto que deviene contradicción cuando la gubernamentalidad neoliberal hace latente su falla sistémica. Lo cual es particularmente relevante en el caso de las personas consideradas con discapacidad, debido a la estrecha relación que existe con la pobreza y la marginalidad.

El Estado articula el campo de inteligibilidad, un dispositivo de múltiples hebras, cuyos haces de luz visibilizan a la discapacidad a partir de diversas tecnologías, dirigiendo su accionar hacia la formación de un Régimen de Verdad: organiza el saber y lo pone a disposición, crea instancias de mediación institucional como la SENADIS, abre espacios de acción y decisión para la población interpelada, promulga leyes para facilitar la prevención, rehabilitación y acceso equitativo a las oportunidades de la sociedad. Sin embargo, aquellos que quedan al margen de las instancias dispuestas, aun cuando participan del mismo campo de inteligibilidad sobre la discapacidad, es decir se pueden identificar a partir del saber sobre sí mismo elaborado, el no estar inscritos en los registros o la imposibilidad de acceder a los servicios de atención estatales a causa de la cobertura insuficiente y los recursos escasos presupuestados, queda a merced de sus posibilidades individuales de acceso a la esfera privada de servicios. Considerando el factor pobreza, esto significa en lo efectivo la continuidad de su condición de exclusión.

Con esto no se contradice el objetivo de la ley de acceso igualitario a las oportunidades. El Estado crea un aparataje institucional y organiza la plataforma para que dicho acceso ocurra. No obstante, debido a la llamada falla sistémica, aquí interviene el factor desde donde el Estado en su nuevo rol gubernamental solo puede asegurar ese servicio a una reducida parte de la población, aquella necesaria para evitar los desbordes de control.

Las subjetividades que se enmarcan en esta tensión, sufren de la frustración de las expectativas de inclusión, pudiendo solo acceder a los resquicios de subsistencias más precarizados como lo son el contrato de trabajo o la dependencia de terceros. La calidad de vida y la inclusión quedan como vestigios de un relato inalcanzable, que sin embargo está ahí, como parte de aquello que define sus posibilidades, un campo de inteligibilidad a partir del cual solo es visible un cuerpo arrojado como residuo que la maquinaria gubernamental deja morir. 


\section{BiBLIOGRAFÍA}

Agamben, G. (2004) Estado de excepción. Homo sacer, II, I. Buenos Aires, Adriana Hidalgo editora.

Barnes, C. (2009) Un Chiste malo: ¿rehabilitar a las personas con discapacidad en una sociedad que discapacita? En Brogna, P. (Comp.)Visiones y Revisiones de la Discapacidad. México D.F. Fondo de Cultura Económica. (101-123)

Brogna, P. (Comp.)Visiones y Revisiones de la Discapacidad. México D.F. Fondo de Cultura Económica (212-235)

Butler, J. (2002) Cuerpos que importan. Sobre los límites materiales y discursivos del 'sexo'. Buenos Aires. Paidós

Camargo, R. (2007) Del "Crecimiento con Equidad" al "Sistema de Protección Social": La Matriz Ideológica del Chile Actual (19902007) En: Revista de Sociología 27/2007 CHILE HOY. 9-31

Casado, D. (2001) Conceptos sobre la discapacidad. Apuntes. En Boletín del Real Patronato sobre Discapacidad (50) 5-13

Deleuze, G. (1995) Conversaciones 1972-1990. Edición electrónica de www.philosophia.cl / Escuela de Filosofía Universidad ARCIS

De Marinis, P. (1999) "Gobierno, gubernamentalidad, Foucault y los anglofoucaultianos" En Fernando García Selgas y Ramón Ramos Torre (Comps.), Globalización, riesgo, reflexividad. Tres temas de la teoría social contemporánea. Madrid. Centro de Investigaciones Sociológicas.

Du gay P., y Hall, S. (Comps.), (2003) Cuestiones de identidad cultural Buenos Aires: Amorrortu

Duschatzky, S y Skliar, C (2000) "La diversidad bajo sospecha. Reflexiones sobre los discursos de la diversidad y sus implicancias educativas" Cuadernos de Pedagogía Rosario No 7

Egea, C. Sarabia, A. (2001) "Clasificaciones de la OMS sobre discapacidad". En: Boletín del Real Patronato sobre Discapacidad (50) $15-30$

Ferreira, M. Ferrante, C. (2009) Cuerpo y Discapacidad: La normalización Médica como Lógica de la dominación. Congreso: El cuerpo, sujeto y objeto de las ciencias humanas y sociales, Instituto Milá y Fonta-nals - CISIC; Barcelona, 28-31 enero 2009; $7 \mathrm{pp}$.

FONADIS (2004) Primer Estudio Nacional de la Discapacidad e Informes Regionales

Foucault, M. (1992) b Nietzsche la genealogía y la historia. Madrid: La Piqueta

(2000) Defender la Sociedad. Buenos Aires: Fondo de Cultura Económica

(2002) Vigilar y Castigar. Nacimiento de la prisión Buenos Aires: Siglo XXI Editores

(2004) Seguridad territorio y población. Buenos Aires, Fondo de Cultura Económica.

Goffman, E. (2006) Estigma. La identidad deteriorada. Amorrortu Editores. Bs As
Grinberg S. (2008) Educación y Poder en el siglo XXI. Gubernamentalidad y pedagogía en las sociedades de gerenciamiento. Argentina Ed. Miño y Dávila

Hardt, M. y Negri, A. (2005) Imperio. Barcelona: Paidós

Landa, M. Marengo, L. (2010) Devenir empresa: el nuevo capitalismo y sus tramas de sujeción. En Actuel Marx Intervenciones (9) 161-182

Le Breton (2002) La Sociología del Cuerpo. Buenos Aires: Nueva Visión

Lemm, V. (Editora) (2010) Michel Foucault: neoliberalismo y biopolítica, Ediciones Universidad Diego Portales

Martínez Posada, J (2010) La universidad productora de productores: entre biopolítica y subjetividad. Bogotá: Universidad de la Salle

Miller, P. Rose, N. (1992) Political Power beyond the State: Problematics of Government. En: The British Journal of Sociology 43 (2) $173-205$

Oliver, M. (1998) ¿Una sociología de la discapacidad o una sociología discapacitada? En: Len Barton (Comp.) Discapacidad y sociedad (39-59) Madrid: Ediciones Morata

Organización Mundial de la Salud (2001) Clasificación internacional del funcionamiento, de la discapacidad y de la salud. CIF. Extraído el 15 de noviembre del 2013 de:

http://conadis.salud.gob.mx/descargas/pdf/Clasificacion-CIF.pdf

Ortuzar, D. (2009) Políticas del Cuerpo en la Discapacidad: Retoricas de la Rehabilitación en Chile. Intersticios: Revista Sociológica de Pensamiento Crítico. (Chile) Vol. 3 (1) 67-77

Rose, N. (1997) El gobierno en las democracias liberales "avanzadas" del liberalismo al neoliberalismo. En Archipiélago: Cuadernos de crítica de la cultura, (29) 25-40.

Rose, N. (1999) Powers of freedom: reframing political thought. Cambridge University Press.

Scribano, A. Figari, C. (Comp.) (2009) Cuerpo(s), Subjetividad(es) y Conflicto(s). Hacia una sociología de los cuerpos y las emociones desde Latinoamérica, CLACSO- CICCUS, Buenos Aires.

Virno, P. (2003) Gramática de la multitud. Para un análisis de las formas de vidas contemporáneas. Madrid. Traficantes de sueños

\section{Bibliografía de la Muestra}

Biblioteca del Congreso Nacional (2008). La historia de la Ley 19.284

Biblioteca del Congreso Nacional (2010) Historia de la Ley $N^{\circ}$ 20.422

Establece Normas Para la Plena Integración Social de Personas con Discapacidad, Ley 19.284 (1994)

Establece Normas sobre Igualdad de Oportunidades e Inclusión Social de Personas con Discapacidad, Ley 20.422, (2010)

Aylwin, P. (1992) Mensaje Presidencial 21 de Mayo

Losav, J. Sar, P. (2010) Reflexiones de la ley 19284. Extraído el 2 de mayo del 2013 de:

http://www.discaparadigma.cl/index.php/reflexiones-de-laley-19284.html

Organización de las Naciones Unidas. Convención sobre los Derechos de las Personas con Discapacidad. Extraído el 9 de mayo de 2013 de: http://www.un.org/esa/socdev/enable/documents/ tccconvs.pdf 
Organización de las Naciones Unidas. Las Naciones Unidas y las Personas con Discapacidad: Los primeros cincuenta años. Extraído el 23 de marzo de 2013 de: www.un.org/spanish/esa/social/ disabled/dis50y00.htm

Organización Internacional del Trabajo (OIT) (1983) C159 Sobre la readaptación profesional y el empleo de personas inválidas. Extraído el 9 de mayo de 2013 de: www.ilo.org/dyn/normlex/es/f?p =NORMLEXPUB:12100:0::NO::P12100_ILO_CODE:C159

Fondo Nacional de la Discapacidad (2006) Desarrollo Inclusivo y Discapacidad: Oportunidades y Desafíos. En Seminario Internacional.

Cisternas, M (2006) Estudio sobre Legislación y Política de Discapacidad en Chile. En Fondo Nacional de la Discapacidad: Seminario Internacional. Desarrollo Inclusivo y Discapacidad: Oportunidades y Desafíos.

Jiménez, L. (2006) Hacia un Sistema Nacional de Promoción de la Autonomía Personal y Atención a las Personas en Situación de Dependencia. En Fondo Nacional de la Discapacidad: Seminario
Internacional. Desarrollo Inclusivo y Discapacidad: Oportunidades y Desafíos.

Savedof, W. (2006) Política pública para las Personas con Discapacidad en Chile: Aprendiendo de las Experiencias Internacionales. En Fondo Nacional de la Discapacidad: Seminario Internacional. Desarrollo Inclusivo y Discapacidad: Oportunidades y Desafíos.

Colectivo Palos de Ciego (2011) Declaración pública. Extraído el 10 de mayo de 2013 de:

https://word.office.live.com/wv/WordView. aspx? FBsrc $=$ https \% 3 A \% 2 F\% 2 Fwww.facebook. com\%2Fdownload\%2Ffile_preview.php\%3Fid\%3D30304 4299812291\%26time\%3D1380570083\%26metadata\&ac cess_token $=667463269 \% 3 \mathrm{AAVIEx}-Z \mathrm{~W} y-57 \mathrm{Urc6BDhJsPd}$ OAWjwvNo0DHyZIOx3KxShw\&title=Declaraci\%C3\%B3n+P\% C3\%BAblica.docx 